\title{
MONOTONE OPERATOR FUNCTIONS, GAPS AND POWER MOMENT PROBLEM
}

\author{
HIROYUKI OSAKA, SERGEI SILVESTROV and JUN TOMIYAMA*
}

(Dedicated to the memory of Gert K. Pedersen)

\begin{abstract}
The article is devoted to investigation of the classes of functions belonging to the gaps between classes $P_{n+1}(I)$ and $P_{n}(I)$ of matrix monotone functions for full matrix algebras of successive dimensions. In this paper we address the problem of characterizing polynomials belonging to the gaps $P_{n}(I) \backslash P_{n+1}(I)$ for bounded intervals $I$. We show that solution of this problem is closely linked to solution of truncated moment problems, Hankel matrices and Hankel extensions. Namely, we show that using the solutions to truncated moment problems we can construct continuum many polynomials in the gaps. We also provide via several examples some first insights into the further problem of description of polynomials in the gaps that are not coming from the truncated moment problem. Also, in this article, we deepen further in another way into the structure of the classes of matrix monotone functions and of the gaps between them by considering the problem of position in the gaps of certain interesting subclasses of matrix monotone functions that appeared in connection to interpolation of spaces and in a proof of the Löwner theorem on integral representation of operator monotone functions.
\end{abstract}

\section{Introduction}

A real-valued continuous function $f: I \rightarrow \mathrm{R}$ is said to be matrix monotone of order $n$ over an interval $I$, if

$$
x \leq y \quad \Rightarrow \quad f(x) \leq f(y)
$$

for any two self-adjoint $n \times n$ matrices $x$ and $y$ with eigenvalues in $I$. We denote the class of all such functions by $P_{n}(I)$. A real-valued continuous function $f: I \rightarrow \mathrm{R}$ on a (non trivial) interval $I \neq \mathrm{R}$ is called operator monotone if the implication (1) holds for any pair of bounded operators $x, y \in B(H)$ on an infinite-dimensional separable Hilbert space $H$ with their spectra in $I$. We denote the class of all operator monotone functions over an interval $I$ by

\footnotetext{
* This work was supported by The Swedish Foundation for International Cooperation in Research and Higher Education (STINT), Crafoord Foundation, The Royal Physiographic Society in Lund and Open Research Center Project for Private Japanese Universities: matching fund from MEXT, 2004-2008.
}

Received March 12, 2006. 
$P_{\infty}(I)$, or simply by $P_{\infty}$ when the choice of the interval is clear from context. For each positive integer $n$, the proper inclusion $P_{n+1}(I) \subsetneq P_{n}(I)$ holds. This fact has been stated in [3], but the complete proof of this appeared first in [5]. The gaps $P_{n}(I) \backslash P_{n+1}(I)$ between classes of monotone matrix functions were also recently addressed in [10] and [8]. For infinite-dimensional Hilbert space, the set of operator monotone functions on $I$ can be shown to coincide with the intersection

$$
P_{\infty}(I)=\bigcap_{n=1}^{\infty} P_{n}(I)
$$

or in other words a function is operator monotone if and only if it is matrix monotone of order $n$ for all positive integers $n$ [6, Chap. 5, Proposition 5.1.5 (1)].

The proof of non-emptiness of gaps $P_{n}(I) \backslash P_{n+1}(I)$ in [5] is constructive, by exhibiting for each positive integer $n$ an explicit function in the gap. Moreover, for any bounded interval and positive integer $n$, the exhibited in [5] function in the gap $P_{n}(I) \backslash P_{n+1}(I)$ was a polynomial, thus suggesting that there might be more polynomials in the gaps for any bounded interval, hence leading directly to an interesting problem of characterizing such polynomials. For the unbounded interval $(0,+\infty)$ it can be shown that there are no polynomials in the gaps. However, the unbounded interval can be bijectively mapped onto a bounded interval using an operator monotone fractional Möbius transformation with operator monotone inverse, and then any polynomial in the gap over that bounded interval, after proper composition with those fractional Möbius transformations, yields a rational function from the gap over the unbounded interval.

In this paper we address the problem of characterizing polynomials belonging to the gaps $P_{n+1}(I) \subsetneq P_{n}(I)$ for bounded intervals $I$. We show that solution of this problem is closely linked to solution of truncated moment problems, Hankel matrices and Hankel extensions. Namely, we show that using the solutions to truncated moment problems we can construct continuum many polynomials in the gaps. We also provide via several examples some first insights into the further problem of description of polynomials in the gaps that are not coming from the truncated moment problem.

Also, in this article, we deepen further in another way into the structure of the classes $P_{n}$ and of the gaps by considering a certain interesting subclass of functions inside $P_{n}$. This class of functions, denoted by $\mathscr{M}_{n}((0,+\infty))$, has been defined in [14], as consisting of real-valued functions $h$ on $(0, \infty)$ such that for $a_{j} \in \mathrm{R}, \lambda_{j}>0$ and $j=1, \ldots, 2 n$ the following implication holds:

$$
\left(\sum_{j=1}^{2 n} a_{j} \frac{t \lambda_{j}-1}{t+\lambda_{j}} \geq 0 \text { for } t>0, \sum_{j=1}^{2 n} a_{j}=0\right) \Rightarrow\left(\sum_{j=1}^{2 n} a_{j} h\left(\lambda_{j}\right) \geq 0\right) .
$$


It was shown in [14] that

$$
P_{n+1}((0,+\infty)) \subseteq \mathcal{M}_{n}((0,+\infty)) \subseteq P_{n}((0,+\infty))
$$

for any positive integer $n$, and so $P_{\infty}=\bigcap_{n=1}^{\infty} P_{n}((0,+\infty))=\bigcap_{n=1}^{\infty} \mathscr{M}_{n}((0$, $+\infty)$ ). In [14], an explicit example, showing that $P_{2} \backslash \mathcal{M}_{2} \neq \emptyset$, has been pointed out, thus particularly implying that $P_{2}((0,+\infty)) \backslash P_{3}((0,+\infty)) \neq \emptyset$. Proving that $P_{n}((0,+\infty)) \backslash \mathscr{M}_{n}((0,+\infty)) \neq \emptyset$ and $\mathscr{M}_{n}((0,+\infty)) \backslash P_{n+1}((0,+\infty))$ $\neq \varnothing$ for an arbitrary $n$ is still an open problem. The unbounded interval $(0,+\infty)$ is a union of inclusion increasing set of bounded intervals $(0,+\infty)=$ $\cup_{a>0}(0, a)$. In this article we consider the classes of functions $\mathscr{M}_{n}(I)$ on the bounded intervals. The definition is the same up to just replacing $(0,+\infty)$ by the bounded interval $I$. The content of the class $\mathscr{M}_{n}(I)$ differs from $\mathcal{M}_{n}((0,+\infty))$. However, we provide in this article a proof that the inclusions

$$
P_{n+1}(I) \subseteq \mathcal{M}_{n}(I) \subseteq P_{n}(I)
$$

hold even for any bounded interval of the form $(0, a)$ or $(0, a]$ and all positive integers $n$. Therefore, we can conclude that $\bigcap_{n=1}^{\infty} \mathcal{M}_{n}(I)=P_{\infty}(I)$. The problem of proving or disproving the existence of the non-empty gap $P_{n}(I) \backslash \mathscr{M}_{n}(I)$ is also an open problem both for the bounded interval $I$ and for $(0,+\infty)$. However, while the example of function in the gap $P_{2}((0,+\infty)) \backslash \mathscr{M}_{2}((0,+\infty))$ constructed in [14] is non-polynomial due to lack of polynomials and also seems to be difficult to extend to an arbitrary $n$, in the case of the bounded interval, we show in this article how to construct explicitly infinitely many polynomials in the gap $P_{n}(I) \backslash P_{n+1}(I)$ for any $n$. Thus a natural problem is to describe position of these polynomials with respect to the gaps $P_{n}(I) \backslash \mathscr{M}_{n}(I)$ and $\mathscr{M}_{n}(I) \backslash P_{n+1}(I)$. We succeeded to investigate this problem for the polynomial in the gap constructed in [5] for $n=2,3,4,5$.

\section{Polynomial Monotone Matrix Functions}

Proposition 2.1. The only polynomials belonging to the class $P_{l}([0,+\infty))$ for an integer $l>1$ are polynomials of the form $a t+b$ where $a \geq 0$.

Proof. Let $p_{n}(t)=\sum_{j=0}^{n} a_{j} t^{n-j}$ be a polynomial in $P_{l}([0,+\infty))$ with $a_{0} \neq 0$. Then for any $C, D \in M_{l}$ such that $0 \leq C \leq D$ and any $\lambda>0$ we have $0 \leq \lambda C \leq \lambda D$ and hence

$$
\begin{aligned}
& a_{n} I \leq \sum_{j=0}^{n} a_{j}(\lambda C)^{n-j} \leq \sum_{j=0}^{n} a_{j}(\lambda D)^{n-j} \\
& \frac{a_{n}}{\lambda^{n}} I \leq \sum_{j=0}^{n} a_{j} \lambda^{-j} C^{n-j} \leq \sum_{j=0}^{n} a_{j} \lambda^{-j} D^{n-j}
\end{aligned}
$$


which, after passing to the limit $\lambda \rightarrow+\infty$, yields $0 \leq a_{0} C^{n} \leq a_{0} D^{n}$ implying $0<a_{0}$ and $0 \leq C^{n} \leq D^{n}$. This holds for arbitrary choice of $0 \leq C \leq D$ only if $n=1$, since $f(t)=t^{n} \notin P_{l}([0,+\infty)) \subset P_{2}([0,+\infty))$ when $n>1$ and $l \geq 2$. Thus, $p_{n}(t)=a_{n-1} t+a_{n}$. When $a_{n-1} \neq 0$, from the same argument as before we have $a_{n-1}>0$, which is exactly what had to be proved.

The situation is totally different on the finite intervals. There polynomials of high degree than one can be matrix monotone of order $n$. There is no contradiction here since the transformations between a finite and an infinite interval do not map polynomials into polynomials. Usually a Möbius transformation can be used for this purpose, and in this case the polynomial on a finite interval will be transformed into a rational function on an infinite interval.

Let $g_{n}(t)=t+\frac{1}{3} t^{3}+\cdots+\frac{1}{2 n-1} t^{2 n-1}$, where $n$ is some positive integer. In [5] it was proved that there exists $\alpha_{n}>0$ such that $g_{n} \in P_{n}\left(\left[0, \alpha_{n}\right)\right) \backslash$ $P_{n+1}\left(\left[0, \alpha_{n}\right)\right)$, and consequently $f_{n}=g_{n} \circ h_{n} \in P_{n} \backslash P_{n+1}$, where $h_{n}(t)$ is the Möbius transformation $h_{n}(t)=\frac{\alpha_{n} t}{1+t}$, operator monotone on $[0, \infty)$, with the inverse $h_{n}^{\circ(-1)}(t)=\frac{t}{\alpha_{n}-t}$ operator monotone on $\left[0, \alpha_{n}\right)$.

Note that two compact intervals can be however mapped to each other with some polynomial of degree one $\alpha t+\beta$ with $\alpha>0$, an operator monotone function on any interval. Namely, the bounded interval with end points $u_{1}<$ $v_{1}$ is mapped to the bounded interval with end points $u_{2}<v_{2}$ by the map $h(t)=\frac{v_{2}-u_{2}}{v_{1}-u_{1}} t+\frac{u_{2} v_{1}-v_{2} u_{1}}{v_{1}-u_{1}}$, with the composition inverse

$$
h^{\circ(-1)}(t)=\frac{v_{1}-u_{1}}{v_{2}-u_{2}} t-\frac{u_{2} v_{1}-v_{2} u_{1}}{v_{2}-u_{2}}
$$

which are both operator monotone since $\frac{v_{2}-u_{2}}{v_{1}-u_{1}}>0$ and $\frac{v_{1}-u_{1}}{v_{2}-u_{2}}>0$. The type of the interval with respect to the inclusion or exclusion of the end points is preserved by this map. Moreover, this map transforms polynomials matrix monotone of order $n$ on one interval into polynomials of the same degree and matrix monotone of order $n$ on the other interval. In particular, $[0, a)$ is transformed to $[u, v)$ by the map $h(t)=\frac{v-u}{a} t+u$ with the composition inverse $h^{\circ(-1)}(t)=\frac{a}{v-u} t-\frac{a u}{v-u}$. The interval $[0, a)$ is mapped to the interval $[0, b)$ by the map $h(t)=\frac{b}{a} t$ with the composition inverse $h^{\circ(-1)}(t)=\frac{a}{b} t$. The interval $[-1,1]$ is mapped to the interval $[u, v]$ by the map $h(t)=\frac{v-u}{2} t+\frac{v+u}{2}$ with the composition inverse $h^{\circ(-1)}(t)=\frac{2}{v-u} t-\frac{v+u}{v-u}$. The interval $[-1,1]$ is mapped to the interval $[0, a]$ by the map $h(t)=\frac{a}{2} t+\frac{a}{2}$ with the composition inverse $h^{\circ(-1)}(t)=\frac{2}{a} t-1$. Keeping these considerations on maps of the intervals in mind, we will work on the intervals containing 0 or other intervals convenient for the proofs, making clear from our statements or by specially pointing out when the choice of the interval is not essential. 
We will make use of the following conditions concerned with $n$-monotonicity of functions on an interval [3], restricting formulation to the functions which are infinitely differentiable, which is suited to our considerations. For every such function and every positive integer $n$ define the matrix $M_{n}(f ; t)=\left(\frac{f^{(i+j-1)}(t)}{(i+j-1) !}\right)_{i, j=1}^{n}$. If $f \in P_{n}((a, b))$ for $n \geq 2$, then $M_{n}(f ; t) \geq 0$ and $f^{(2 n-3)}(t)$ is convex on $(a, b)$, by [3, Theorem VI, Ch. VII]. Conversely, if $M_{n}(f ; t) \geq 0$ and the derivative $f^{(2 n-3)}(t)$ is positive and convex, then $f \in P_{n}((a, b))$, by [3, Theorem V, Ch. VIII]).

THEOREM 2.2. Let I $\subset \mathrm{R}$ be a bounded interval on the real line. There are no polynomials of degree $1<\operatorname{deg}(f)<2 n-1$ in the class $P_{n}(I)$, and there exists a polynomial $f$ of any order $\operatorname{deg}(f) \geq 2 n-1$ in $P_{n}(I)$. Any polynomial in $P_{n}(I)$ of degree $\operatorname{deg}(f)=2 n-1$ or $\operatorname{deg}(f)=2 n$ belongs to the gap $f \in P_{n}(I) \backslash P_{n+1}(I)$.

Proof. Let $f(t)=c+\sum_{j=0}^{k-1} b_{j} t^{j+1}$, where $b_{k-1} \neq 0$ and $1<k=$ $\operatorname{deg}(f)<2 n-1$. We consider two cases, of odd and even $k$. Let $k=2 l$ with $l \geq 1$. Then

$$
M_{l+1}(f ; 0)=\left(\begin{array}{ccccc}
b_{0} & b_{1} & \ldots & b_{l-1} & b_{l} \\
b_{1} & \ldots & \ldots & b_{l} & b_{l+1} \\
\vdots & \vdots & \vdots & \vdots & \vdots \\
b_{l-1} & b_{l} & \ldots & b_{2 l-2} & b_{2 l-1} \\
b_{l} & b_{l+1} & \ldots & b_{2 l-1} & 0
\end{array}\right)
$$

Since $k<2 n-1$, the matrix $M_{l+1}(f ; 0)$ is contained as the principle upper left corner submatrix of $M_{n}(f ; 0)$. Because

$$
\operatorname{det}\left(\begin{array}{cc}
b_{k-2} & b_{k-1} \\
b_{k-1} & 0
\end{array}\right)=-\left(b_{k-1}\right)^{2}<0,
$$

the matrix $M_{n}(f ; 0)$ is not positive definite and therefore $f \notin P_{n}(I)$. In the odd case, that is for $k=2 l-1, l \geq 2$ and $b_{2 l-2} \neq 0$, one has

$$
M_{l+1}(f ; 0)=\left(\begin{array}{cccccc}
b_{0} & b_{1} & \ldots & b_{l-2} & b_{l-1} & b_{l} \\
b_{1} & b_{2} & \ldots & b_{l-1} & b_{l} & b_{l+1} \\
\vdots & \vdots & \vdots & \vdots & \vdots & \vdots \\
b_{l-2} & b_{l-1} & \ldots & b_{2 l-4} & b_{2 l-3} & b_{2 l-2} \\
b_{l-1} & b_{l} & \ldots & b_{2 l-3} & b_{2 l-2} & 0 \\
b_{l} & b_{l+1} & \ldots & b_{2 l-2} & 0 & 0
\end{array}\right) .
$$

Suppose that $f \in P_{n}(I)$ and hence $M_{n}(f ; 0) \geq 0$. Then $b_{k-1}=b_{2 l-2}>0$, since $M_{n}(f ; 0) \geq 0$ and since $b_{k-1}=b_{2 l-2} \neq 0$ as the highest coefficient of 
the polynomial $f$. Thus

$$
\operatorname{det}\left(\begin{array}{ccc}
b_{2 l-4} & b_{2 l-3} & b_{2 l-2} \\
b_{2 l-3} & b_{2 l-2} & 0 \\
b_{2 l-2} & 0 & 0
\end{array}\right)=-\left(b_{2 l-2}\right)^{3}<0 .
$$

Hence the matrix $M_{n}(f ; 0)$ is not positive semi-definite which contradicts to the assumption $f \in P_{n}(I)$. Therefore $f \notin P_{n}(I)$.

In [5] it was proved that for any positive integer $n$ there exists $\alpha_{n}>0$ such that $g_{n}(t)=t+\frac{1}{3} t^{3}+\cdots+\frac{1}{2 n-1} t^{2 n-1} \in P_{n}\left(\left[0, \alpha_{n}\right)\right) \backslash P_{n+1}\left(\left[0, \alpha_{n}\right)\right)$. Consequently, if $I=[u, v)$, then $\tilde{g}_{n}=g_{n} \circ h^{\circ(-1)} \in P_{n}([u, v)) \backslash P_{n+1}([u, v))$ where $\tilde{g}_{n}$ is the polynomial of degree $2 n-1$ obtained by composition of $g_{n}$ with the operator monotone affine transformation $h^{\circ(-1)}(t)=\frac{\alpha_{n}}{v-u} t-\frac{\alpha_{n} u}{v-u}$, mapping interval $[u, v)$ onto $\left[0, \alpha_{n}\right)$. In order to show existence of the polynomials of the even degree in the gap, take $p_{n}(t)=t+\frac{1}{3} t^{3}+\cdots+\frac{1}{2 n-1} t^{2 n-1}+a t^{2 n}$. By the first statement of the theorem we have already proved that $p_{n} \notin P_{n+1}([0, \alpha))$ for any $\alpha>0$ since $\operatorname{deg}\left(p_{n}\right)=2 n<2(n+1)-1=2 n+1$. Since $\operatorname{det} M_{n}\left(p_{n} ; 0\right)=$ $\operatorname{det} M_{n}\left(g_{n} ; 0\right)$, there exists $\alpha_{n}^{\prime}>0$ such that $p_{n} \in P_{n}\left(\left[0, \alpha_{n}^{\prime}\right)\right) \backslash P_{n+1}\left(\left[0, \alpha_{n}^{\prime}\right)\right)$. Therefore, the polynomial $p_{n} \circ \tilde{h}^{\circ(-1)}$ of degree $2 n$, obtained by composition with $\tilde{h}^{\circ(-1)}(t)=\frac{\alpha_{n}^{\prime}}{v-u} t-\frac{\alpha_{n}^{\prime} u}{v-u}$, belongs to the gap $P_{n}([u, v)) \backslash P_{n+1}([u, v))$ over the interval $[u, v)$. Since $P_{n}(I) \supset P_{n+k}(I)$ for any $k \geq 1$, there exists a polynomial $f$ of any order $\operatorname{deg}(f) \geq 2 n-1$ in $P_{n}(I)$.

Finally, by the first statement of the theorem, any polynomial of degree $2 n-1$ or $2 n$ does not belong to $P_{n+1}(I)$ since $2 n-1<2 n<2(n+1)-1=$ $2 n+1$, and hence if it is in addition a polynomial from $P_{n}(I)$, then it belongs to the gap $P_{n}(I) \backslash P_{n+1}(I)$.

\section{Truncated Moment Problem and Monotone Matrix Functions}

THeORem 3.1. Let $f(t)=c+b_{0} t+b_{1} t^{2}+\cdots+b_{2 n-2} t^{2 n-1}+b_{2 n-1} t^{2 n}+\cdots$ be a polynomial of degree at least $2 n-1$. Then

(a) $M_{n}(f ; 0)>0$ if and only if there is a Borel measure $\mu$ on $\mathbf{R}$ with at least $n$ points in the support, and such that

$$
b_{k}=\int_{\mathrm{R}} t^{k} d \mu<\infty \quad(0 \leq k \leq 2 n-2) .
$$

Moreover, in this case there exists $\alpha_{n}>0$ such that $f \in P_{n}\left(\left[0, \alpha_{n}\right)\right)$.

(b) If $M_{n}(f ; 0) \geq 0$ but $\operatorname{det} M_{n}(f ; 0)=0$, and $r$ is the smallest positive integer such that $M_{r+1}(f ; 0)$ is not invertible, then there exists a Borel 
measure $\mu$ such that

$$
b_{k}=\int_{\mathrm{R}} t^{k} d \mu<\infty \quad(0 \leq k \leq 2 r-2),
$$

and there exists $\alpha>0$ such that $f \in P_{r}([0, \alpha))$.

Proof. (a) At first we recall that the inequality $M_{n}(f ; 0)>0$ means that the Hankel matrix $M_{n-1}(f ; 0)$ has a positive Hankel extension $M_{n}(f ; 0)$, and hence by [2, Theorem 3.9], this is equivalent to the existence of a Borel measure $\mu$ on $\mathrm{R}$, such that

$$
b_{k}=\int_{\mathrm{R}} t^{k} d \mu<\infty \quad(0 \leq k \leq 2 n-2) .
$$

Suppose that the measure $\mu$ has at least $n$ points in the support and satisfies $b_{k}=\int_{\mathrm{R}} t^{k} d \mu<\infty$ when $0 \leq k \leq 2 n-2$. Take arbitrary $n$ points $t_{1}, \ldots, t_{n}$ in the support of $\mu$. Then $\mu\left(I_{i}\right)>0$ for any family of $n$ non-overlapping open intervals such that $t_{i} \in I_{i}$ for $i=1, \ldots, n$. Choose inside each of these open intervals a closed interval $J_{i}$ such that $t_{i} \in J_{i} \subset I_{i}$ and hence also $\mu\left(J_{i}\right)>0$ for $i=1, \ldots, n$. For any vector $\vec{c}=\left(c_{0}, \ldots, c_{n-1}\right) \in \mathrm{C}^{n}$, the following holds for the quadratic form

$$
\begin{aligned}
\left(M_{n}(f ; 0) \vec{c} \mid \vec{c}\right) & =\sum_{i=0}^{n-1} \sum_{j=0}^{n-1} b_{i+j} c_{j} \overline{c_{i}}=\sum_{i=0}^{n-1} \sum_{j=0}^{n-1} \int_{\mathrm{R}} t^{i+j} d \mu c_{j} \bar{c}_{i} \\
& =\int_{\mathrm{R}}\left|\sum_{i=0}^{n-1} c_{i} t^{i}\right|^{2} d \mu \geq\left|\sum_{i=0}^{n-1} c_{i} \hat{t}_{k}^{i}\right|^{2} \mu\left(J_{k}\right) \geq 0,
\end{aligned}
$$

where $\hat{t}_{k}$ is the minimum point for the continuous function $\left|\sum_{i=0}^{n-1} c_{i} t^{i}\right|^{2}$ on the closed interval $J_{k}$. Therefore, the matrix $M_{n}(f ; 0)$ is at least positive semidefinite. Moreover, since $\mu\left(J_{k}\right)>0$, if $\left(M_{n}(f ; 0) \vec{c} \mid \vec{c}\right)=0$ for some $\vec{c}$, then $\sum_{i=0}^{n-1} c_{i} \hat{t}_{k}^{i}=0$ for all $k=1, \ldots, n$. Since, only the zero polynomial has more roots than its degree, the only possibility for the linear system to hold is when $\vec{c}=\overrightarrow{0}$. Therefore, the matrix $M_{n}(f ; 0)$ is positive definite $\left(M_{n}(f ; 0)>\right.$ $0)$. All elements of this matrix are polynomials and hence determinants of all submatrices are also polynomials, and in particular the determinants of all submatrices with the principal diagonals, consisting from elements of the principal diagonal of $M_{n}(f ; 0)$, are also polynomials and hence are continuous functions on the real line. There are finitely many of them and all of them are positive at $t=0$ due to positive definiteness of $M_{n}(f ; 0)$. Each of these polynomials is then positive on some interval of the form $[0, \alpha)$, and taking 
the interval with smallest positive $\alpha$ yields an interval of this form where the matrix $M_{n}(f ; t)$ is positive. Without providing a way to construct $[0, \alpha)$ one may alternatively argue that since eigenvalues of a matrix depend continuously on each entry of a matrix, there exists a positive $\alpha>0$ such that $M_{n}(f ; t)$ is positive semi-definite on $[0, \alpha)$. Hence $f \in P_{n}([0, \alpha))$ by $[3$, Theorem $\mathrm{V}$, Ch. VIII].

To prove the converse implication assume that $M_{n}(f ; 0)>0$ and let $\mu$ be a measure satisfying $b_{k}=\int_{\mathrm{R}} t^{k} d \mu<\infty$ when $0 \leq k \leq 2 n-2$. Assume contrary to the statement in the theorem, that support of $\mu$ contains less than $n$ points. Let $\left\{t_{1}, \ldots, t_{k}\right\}$, where $k<n$, be the support of $\mu$. Then there exists a non-zero polynomial $p_{n}(t)=c_{0}+c_{1} t+\cdots+c_{n-1} t^{n-1}$ such that $p_{n}\left(t_{i}\right)=0$ when $1 \leq i \leq k$. But then for the vector $\vec{c} \neq 0$ of coefficients of this non-zero polynomial

$$
\left(M_{n}(f ; 0) \vec{c} \mid \vec{c}\right)=\int_{\mathrm{R}}\left|\sum_{i=0}^{n-1} c_{i} t^{i}\right|^{2} d \mu=\sum_{j=1}^{k} \mu\left(t_{j}\right)\left|p_{n}\left(t_{j}\right)\right|^{2}=0 .
$$

This contradicts to the assumption $M_{n}(f ; 0)>0$. Thus $\mu$ must have at least $n$ points in its support.

(b) The existence of the measure such that $b_{k}=\int_{\mathrm{R}} t^{k} d \mu<\infty$ when $0 \leq k \leq 2(r-1)=2 r-2$ follows from [2, Theorem 3.9], and existence of $\alpha>0$ such that $f \in P_{r}([0, \alpha))$ is implied from (a) since $M_{r}(f ; 0)>0$ by definition of $r$.

A Hankel $\operatorname{rank}_{\operatorname{rank}}(\vec{\gamma})$ of $\vec{\gamma}=\left(\gamma_{0}, \ldots, \gamma_{2 k}\right)$ associated to a Hankel matrix $\left(\gamma_{i+j}\right)_{i, j=0}^{k}$ of size $(k+1) \times(k+1)$ is defined as the smallest integer $i$ obeying $1 \leq i \leq k$ and such that $\vec{v}_{i}$ is a linear combination of $\vec{v}_{0}, \ldots, \vec{v}_{i-1}$, where $\vec{v}_{j}=$ $\left(\gamma_{j+l}\right)_{l=0}^{k}$ are column vectors of the matrix, that is, $\left(\gamma_{i+j}\right)_{i, j=0}^{k}=\left(\vec{v}_{0}, \ldots, \vec{v}_{k}\right)$. This is a handy notion which we will use in several examples. According to [2, Proposition 2.2] for a positive semidefinite Hankel matrix the Hankel rank of the defining sequence $\vec{\gamma}$ coincides with the smallest positive integer $l$ such that the principle upper left-hand corner submatrix $\left(\gamma_{i+j}\right)_{i, j=0}^{l}$ of size $(l+1) \times(l+1)$ is not invertible, i.e. has zero determinant, or equivalently this can be rephrased as the largest integer $l$ such that all the submatrices $\left(\gamma_{i+j}\right)_{i, j=0}^{m-1}$ of size $m \times m$ with $1 \leq m \leq l$ are invertible. Thus, in this terminology the integer $r$ used in the part (b) of Theorem 3.1 is exactly the Hankel rank of the sequence $\left\{\gamma_{k}=\frac{f^{(k+1)}(0)}{(k+1) !}\right\}_{k=0}^{2(n-1)}$ corresponding to the matrix $M_{n}(f ; 0)$.

We present now an example $p$ of a polynomial of degree 3 which has determinant of the matrix $M_{2}(p ; t)$ at $t=0$ being zero, but $p \in P_{2}([0, \alpha))$ for some $\alpha>0$. Let $f(t)=t-t^{2}+t^{3}$. Then

$$
f^{\prime}(t)=1-2 t+3 t^{2}, \quad f^{\prime \prime}(t)=-2+6 t, \quad f^{\prime \prime \prime}(t)=6 .
$$


Hence

$$
M_{2}(f ; t)=\left(\begin{array}{cc}
1-2 t+3 t^{2} & -1+3 t \\
-1+3 t & 1
\end{array}\right)
$$

Then we have

$$
\operatorname{det}\left(M_{2}(f ; t)\right)=4 t-6 t^{2}=-6\left(t-\frac{1}{3}\right)^{2}+\frac{2}{3} .
$$

If take $\alpha=\frac{2}{3}, f \in P_{2}([0, \alpha))$.

Next we will show that a polynomial $p$ of degree 5 does not belong to $P_{3}([0, \alpha))$ for any $\alpha>0$ when $\operatorname{rank} M_{3}(p ; 0)=2$ and $\operatorname{rank}_{h}(\vec{\gamma})=$ $\operatorname{rank}_{h}\left(b_{0}, b_{1}, b_{2}, b_{3}, b_{4}\right)=1$, where $p(t)=b_{0} t+b_{1} t^{2}+b_{2} t^{3}+b_{3} t^{4}+b_{4} t^{5}$. Let

$$
M_{3}(p ; 0)=\left(\begin{array}{lll}
b_{0} & b_{1} & b_{2} \\
b_{1} & b_{2} & b_{3} \\
b_{2} & b_{3} & b_{4}
\end{array}\right)
$$

Since $\operatorname{rank}_{h}(\vec{\gamma})=\operatorname{rank}_{h}\left(b_{0}, b_{1}, b_{2}, b_{3}, b_{4}\right)=1$, we have

$$
\begin{aligned}
& b_{1}=\lambda b_{0} \\
& b_{2}=\lambda b_{1}=\lambda^{2} b_{0} \\
& b_{3}=\lambda b_{2}=\lambda^{3} b_{0}
\end{aligned}
$$

for some $\lambda \in \mathrm{R}$. Since $\operatorname{rank} M_{3}(p ; 0)=2, b_{0}>0$, and we may assume that $b_{0}=1$. Hence we consider

$$
p(t)=t+\lambda t+\lambda^{2} t^{3}+\lambda^{3} t^{4}+c t^{5} .
$$

for any $c \geq 0$.

Proposition 3.2. Let $p(t)=b_{0} t+b_{1} t^{2}+b_{2} t^{3}+b_{3} t^{4}+b_{4} t^{5}$. Suppose that $\operatorname{rank} M_{3}(p ; 0)=2$ and $\operatorname{rank}_{h}(\vec{\gamma})=\operatorname{rank}_{h}\left(b_{0}, b_{1}, b_{2}, b_{3}, b_{4}\right)=1$. Then there exists no $\alpha>0$ that satisfy $p \in P_{3}([0, \alpha))$.

Proof. From the above argument we assume that

$$
p(t)=t+\lambda t+\lambda^{2} t^{3}+\lambda^{3} t^{4}+c t^{5} .
$$

For the matrix

$$
M_{3}(p ; t)=\left(\begin{array}{ccc}
1+2 \lambda t+3 \lambda^{2} t^{2}+4 \lambda^{3} t^{3}+5 c t^{4} & \lambda+3 \lambda^{2} t+6 \lambda^{3} t^{2}+10 c t^{3} & \lambda^{2}+4 \lambda^{3} t+10 c t^{2} \\
\lambda+3 \lambda^{2} t+6 \lambda^{3} t^{2}+10 c t^{3} & \lambda^{2}+4 \lambda^{3} t+10 c t^{2} & \lambda^{3}+5 c t \\
\lambda^{2}+4 \lambda^{3} t+10 c t^{2} & \lambda^{3}+5 c t & c
\end{array}\right),
$$




$$
\begin{array}{r}
\operatorname{det} M_{3}(p ; t)=30 \lambda^{4} t^{2} c-15 c^{2} t^{2}-15 \lambda^{8} t^{2}-30 \lambda^{5} c t^{3}+50 \lambda c^{2} t^{3} \\
-20 \lambda^{9} t^{3}-210 \lambda^{3} c^{2} t^{5}-105 \lambda^{6} c t^{4}-175 c^{3} t^{6} \\
=t^{2}\left(30 \lambda^{4} c-15 c^{2}-15 \lambda^{8}\right)-30 \lambda^{5} c t^{3}+50 \lambda c^{2} t^{3} \\
-20 \lambda^{9} t^{3}-210 \lambda^{3} c^{2} t^{5}-105 \lambda^{6} c t^{4}-175 c^{3} t^{6}
\end{array}
$$

with $30 \lambda^{4} c-15 c^{2}-15 \lambda^{8}=15\left(2 \lambda^{4} c-c^{2}-\lambda^{8}\right) \leq 15\left(2 \lambda^{4} c-2 \lambda^{4} c\right)=0$, where the equality holds when $c=\lambda^{4}$.

For $c \neq \lambda^{4}$, the coefficient of $t^{2}$ is negative, and there exists $\alpha>0$, such that det $M_{3}(p ; t)<0$ for any $t \in[0, \alpha)$. If $c=\lambda^{4}$, then

$$
\begin{aligned}
\operatorname{det} M_{3}(p ; t) & =-210 \lambda^{3} c^{2} t^{5}-105 \lambda^{6} c t^{4}-175 c^{3} t^{6} \\
& =-105 \lambda^{10} t^{4}-210 \lambda^{11} t^{5}-175 \lambda^{12} t^{6} .
\end{aligned}
$$

Since $-105 \lambda^{10}<0$, there exists $\alpha>0$ such that det $M_{3}(p ; t)<0$ for any $t \in[0, \alpha)$. Hence there exists no $\alpha>0$ such that $p \in P_{3}([0, \alpha))$.

As another example consider $p(t)=\frac{1}{2} t+t^{2}+\frac{1}{2} t^{3}+t^{4}+\frac{1}{2} t^{5}$. Then $\operatorname{det} M_{3}(p ; 0)=0$ and $M_{3}(p ; 0)$ has rank 2. Note that $\operatorname{rank}_{h}\left(\frac{1}{2}, 1, \frac{1}{2}, 1, \frac{1}{2}\right)=2$. Therefore, the situation is different in the previous proposition. Since

$$
M_{3}(p ; t)=\left(\begin{array}{ccc}
\frac{1}{2}+2 t+\frac{3}{2} t^{2}+2 t^{3}+\frac{5}{2} t^{4} & 1+\frac{3}{2} t+6 t^{2}+5 t^{3} & \frac{1}{2}+4 t+5 t^{2} \\
1+\frac{3}{2} t+6 t^{2}+5 t^{3} & \frac{1}{2}+4 t+5 t^{2} & 1+\frac{5}{2} t \\
\frac{1}{2}+4 t+5 t^{2} & 1+\frac{5}{2} t & \frac{1}{2}
\end{array}\right),
$$

we have

$$
\operatorname{det} M_{3}(p ; t)=\frac{9}{2} t+\frac{63}{8} t^{2}-\frac{27}{2} t^{3}-\frac{93}{2} t^{4}-45 t^{5}-\frac{175}{8} t^{6} .
$$

Hence there exists $\alpha>0$ such that $p \in P_{3}([0, \alpha))$.

The previous results and examples imply the following theorem which is concerned with catching conditions for a more precise determination of position of a given function with respect to the decreasing sequences of inclusions for the classes of matrix monotone functions.

Theorem 3.3. Let $0 \in[0, \alpha)$ and let $f$ be a polynomial such that $f \in$ $P_{n}([0, \alpha))$.

(1) If $f \in P_{n+1}([0, \alpha))$, then there exists a Borel measure $\mu$ such that $b_{k}=\int_{\mathrm{R}} t^{k} d \mu$ for $0 \leq k \leq 2 n-1$.

(2) If $f \in P_{n+1}([0, \alpha))$ and $M_{n+1}(f ; 0)>0$, then there exists a Borel measure $\mu$ such that $b_{k}=\int t^{k} d \mu$ for $0 \leq k \leq 2 n$; 
(3) Let $f \in P_{n}([0, \alpha))$ and let $r$ be the smallest number such that the submatrix $M_{r+1}(f ; 0)$ is not invertible. If $r<\operatorname{rank}\left(M_{n}(f ; 0)\right)$, then $f \notin P_{n+1}([0, \alpha))$.

Proof. (1) If $f \in P_{n+1}([0, \alpha))$, then $M_{n+1}(f ; 0) \geq 0$. Hence $M_{n}(f ; 0)$ has a positive semidefinite Hankel extension, and thus according to [2, Theorem 3.1] there exists a Borel measure $\mu$ such that $b_{k}=\int_{\mathrm{R}} t^{k} d \mu$ for $0 \leq k \leq$ $2 n-1$.

(2) If $f \in P_{n+1}([0, \alpha))$ and moreover $M_{n+1}(f ; 0)>0$, then the existence of a Borel measure $\mu$ such that $b_{k}=\int_{\mathrm{R}} t^{k} d \mu$ for $0 \leq k \leq 2 n-1$ is already secured by 1) and then the fact that the next coefficient $b_{2 n}$ may also be determined by the moment $b_{2 n}=\int_{\mathrm{R}} t^{2 n} d \mu$, as claimed in the theorem, follows from the statement (a) of Theorem 3.1, since in this case $2(n+1)-2=2 n$.

(3) If $f \in P_{n+1}([0, \alpha))$, then $M_{n}(f ; 0)$ has positive Hankel extension, and by [2, Theorem 3.9] the ordinary matrix rank of $M_{n}(f ; 0)$ has to be equal to the Hankel rank $r$. Hence, if this equality does not hold, then $f \notin P_{n+1}([0, \alpha))$.

\section{Rank and the Hadamard product}

In this section we treat the rank comparison problem between an $n \times n$ matrix $A$ and $A \bullet D$, where $\bullet$ means the Hadamard product of the matrix $A$ with another matrix $D$, and then we show that these matrix results are useful for understanding of the transformations of matrix monotonicity properties of functions when changing from one interval to another.

Let $A$ be an $n \times n$ matrix

$$
\left(\begin{array}{ccc}
a_{11} & \cdots & a_{1 n} \\
\vdots & & \vdots \\
a_{n 1} & \cdots & a_{n n}
\end{array}\right) .
$$

By $A(k)$ for $(1 \leq k \leq n)$ we denote the $k \times k$ left upper corner matrix, that is,

$$
A(k)=\left(\begin{array}{ccc}
a_{11} & \cdots & a_{1 k} \\
\vdots & & \vdots \\
a_{k 1} & \cdots & a_{k k}
\end{array}\right)
$$

For $n \times n$ matrices $A=\left(a_{i j}\right)$ and $D=\left(d_{i j}\right)$ we write

$$
A \bullet D=\left(a_{i j} d_{i j}\right) \text {. }
$$

Lemma 4.1 ([13, Theorem 5.1.7]). Let $A$ and $B$ be two $n \times n$ matrices. Then

$$
\operatorname{rank}(A \bullet B) \leq(\operatorname{rank}(A))(\operatorname{rank}(B)) .
$$


For an $n \times n$ matrix $A$ with real eigenvalues, we denote by $\lambda_{\min }(A)$ the minimal eigenvalue of $A$, and by $\lambda_{\max }(A)$ the maximal eigenvalue of $A$.

Lemma 4.2 ([13, Theorem 5.3.4]). Let $A$ and $B$ be two $n \times n$ positive semidefinite matrices. Then any eigenvalue $\lambda(A \bullet B)$ of $A \bullet B$ satisfies

$$
\begin{aligned}
\lambda_{\min }(A) \lambda_{\min }(B) & \leq\left(\min _{1 \leq i \leq n}\left(a_{i i}\right)\right) \lambda_{\min }(B) \leq \lambda(A \bullet B) \\
& \leq\left(\max _{1 \leq i \leq n}\left(a_{i i}\right)\right) \lambda_{\max }(B) \leq \lambda_{\max }(A) \lambda_{\max }(B)
\end{aligned}
$$

Using the above two Lemmas we will show the following result.

Proposition 4.3. Let $A$ and $D$ be positive semidefinite $n \times n$ matrices. Suppose that $D=\left(\alpha^{i+j-1}\right)_{i, j=1}^{n}$ and $\alpha>0$. Then for $1 \leq k \leq n$, the matrix $A(k)$ is invertible if and only if $(A \bullet D)(k)$ is invertible.

Proof. Note that $\operatorname{rank}(D(k))=1$ for all $1 \leq k \leq n$. Suppose that $A(k)$ is invertible. Then since $(A \bullet D)(k)=A(k) \bullet D(k)=D(k) \bullet A(k)$, from Lemma 4.2,

$$
\lambda((A \bullet D)(k)) \geq\left(\min _{1 \leq i \leq n}\left\{d_{i i}\right\}\right) \lambda_{\min }(A(k))>0 .
$$

Since any eigenvalue of $(A \bullet D)(k)$ is positive, $(A \bullet D)(k)$ is invertible.

Conversely, suppose that $(A \bullet D)(k)$ is invertible. From Lemma 4.1

$$
k=\operatorname{rank}((A \bullet D)(k)) \leq \operatorname{rank}(A(k)) \operatorname{rank}(D(k)) \leq \operatorname{rank}(A(k)) .
$$

Hence $\operatorname{rank}(A(k))=k$, and $A(k)$ is invertible.

Corollary 4.4. Let $A$ be a positive semidefinite $n \times n$ matrix and define the $n \times n$ matrix $D=\left(\alpha^{i+j-1}\right)_{i, j=1}^{n}$ with $\alpha \neq 0$. Then for $1 \leq k \leq n, A(k)$ is invertible if and only if $(A \bullet D)(k)$ is invertible.

Proof. If $\alpha>0$, then $D$ is a positive semidefinite matrix from the elementary calculation. So, the conclusion follows from the previous proposition.

For $\alpha<0$, the matrix $-D$ is positive semidefinite. Since $-(A \bullet D)=$ $A \bullet(-D)$ and $\operatorname{rank}(A \bullet D)=\operatorname{rank}(-(A \bullet D))$, we get the conclusion.

The presented results on the rank for Hadamard product of matrices are quite useful when attempting to describe how the classes $P_{n}(I)$ are related to each other for different intervals.

Let $f(t)=b_{0} t+b_{1} t^{2}+\cdots+b_{2 n-2} t^{2 n-1}$. The interval $[0, a)$ is transformed bijectively to $[u, v)$ by the operator monotone affine mapping $h(t)=\frac{v-u}{a} t+u$ with the operator monotone composition inverse $h^{\circ(-1)}(t)=\frac{a}{v-u} t-\frac{a u}{v-u}$. 
Then applying the chain rule and the affine form of $h$ and $h^{\circ(-1)}$ we have that $M_{n}\left(f \circ h^{\circ(-1)} ; 0\right)=M_{n}(f ; a) \bullet\left(\left(\left(\frac{a}{v-u}\right)^{i+j-1}\right)_{i, j=1}^{n}\right)$. Hence the ranks of $M_{n}\left(f \circ h^{\circ(-1)} ; 0\right)$ and of $M_{n}(f ; a)$ coincide according to Corollary 4.4. Thus if $u(t)=c_{0}(t-a)+\cdots+c_{2 n-1}(t-a)^{2 n-1}$ on the interval $[u, v)$, and correspondingly $u \circ h^{\circ(-1)}(t)=b_{0} t+\cdots+b_{2 n-2} t^{2 n-1}$ on the interval $[0, \alpha)$, then there exists a measure $\mu$ such that $b_{k}=\int t^{k} d \mu$ for $0 \leq k \leq 2 n-2$ if and only if there exists a measure $\tilde{\mu}$ such that $c_{k}=\int t^{k} d \tilde{\mu}$ for $0 \leq k \leq 2 n-2$. Therefore, in this sense there is a correspondence between the structure of those polynomials in $P_{n}([u, v))$ and $P_{n}([0, \alpha))$.

\section{The characterization of operator monotone function over $[0, a)$}

Let $I=[0, a)$ for $a>0$.

Definition 5.1. Let $\mathscr{M}_{n}(I)$ be the class of functions such that $f \in \mathscr{M}_{n}(I)$ if for all $a_{k} \in \mathrm{R}, \lambda_{k} \in(0, a)$ for $1 \leq k \leq 2 n$

$$
\left\{\begin{array}{l}
\sum_{k=1}^{2 n} a_{k} \frac{\lambda_{k}}{t+\lambda_{k}} \geq 0 \quad \text { for } t>0 \\
\sum_{k=1}^{2 n} a_{k}=0
\end{array}\right.
$$

implies that

$$
\sum_{k=1}^{2 n} a_{k} f\left(\lambda_{k}\right) \geq 0
$$

The above class $\mathscr{M}_{n}(I)$ is a finite interval version of the class $\mathscr{M}_{n}$ in [14].

REMARK 5.2. Since for $t>0$ and $\sum_{k=1}^{2 n} a_{k}=0$,

$$
\sum_{k=1}^{2 n} a_{k} \frac{\lambda_{k} t-1}{t+\lambda_{k}}=\left(t+\frac{1}{t}\right) \sum_{k=1}^{2 n} a_{k} \frac{\lambda_{k}}{t+\lambda_{k}},
$$

$f \in \mathscr{M}_{n}(I)$ if and only if

$$
\left\{\begin{array}{l}
\sum_{k=1}^{2 n} a_{k} \frac{\lambda_{k} t-1}{t+\lambda_{k}} \geq 0 \quad \text { for } t>0 \\
\sum_{k=1}^{2 n} a_{k}=0
\end{array}\right.
$$


implies that

$$
\sum_{k=1}^{2 n} a_{k} f\left(\lambda_{k}\right) \geq 0
$$

We use the following characterization of functions in $P_{n}(I)$ given in [14].

Lemma 5.3. For $\alpha$ and $x$ in $\mathrm{C}^{n}$ set $\|x\|_{\alpha}=\left(\sum_{k=1}^{n} \alpha_{k}\left|x_{k}\right|^{2}\right)^{\frac{1}{2}}$.

Then $f \in P_{n}(I)$ if and only if for all $n \times n$ unitary $U$ with $\|U\|_{\alpha, \beta} \leq 1$ $\left(\alpha, \beta \in \mathrm{C}^{n} \cap I^{n}\right)$ :

$$
\sum_{k=1}^{n} f\left(\alpha_{k}\right)\left|x_{k}\right|^{2} \geq \sum_{k=1}^{n} f\left(\beta_{k}\right)\left|(U x)_{k}\right|^{2}, \quad \forall x \in \mathrm{C}^{n},
$$

where

$$
\|U\|_{\alpha, \beta}=\sup _{x \in C^{n} \backslash\{0\}} \frac{\|U x\|_{\beta}}{\|x\|_{\alpha}} .
$$

Proof. Let $A$ and $B$ be two hermitian $n \times n$ matrices with eigenvalues contained in $I$. Then

$$
A \geq B \Leftrightarrow \sum_{k=1}^{n} \alpha_{k}\left|x_{k}\right|^{2} \geq \sum_{k=1}^{n} \beta_{k}\left|(U x)_{k}\right|^{2}, \quad \forall x \in \mathbb{C}^{n}
$$

where $x=\left(x_{1}, x_{2}, \ldots, x_{n}\right)^{T}, \alpha_{1}, \alpha_{2}, \ldots, \alpha_{n}$ are eigenvalues for $A, \beta_{1}, \beta_{2}$, $\ldots, \beta_{n}$ eigenvelues for $B$, and $U$ is an appropriate $n \times n$ unitary. Every unitary arises for some choice of $A$ and $B$. Hence we have $f \in P_{n}(I) \Leftrightarrow \forall n \times n$ unitary $U$ with $\|U\|_{\alpha, \beta} \leq 1\left(\alpha, \beta \in \mathrm{C}^{n} \cap I^{n}\right)$

$$
\sum_{k=1}^{n} f\left(\alpha_{k}\right)\left|x_{k}\right|^{2} \geq \sum_{k=1}^{n} f\left(\beta_{k}\right)\left|(U x)_{k}\right|^{2}, \quad \forall x \in \mathrm{C}^{n} .
$$

As for $\mathcal{M}_{n}$ in [14] we have the following fundamental inclusion.

Proposition 5.4. For all $n \in \mathrm{N}$

$$
P_{n+1}(I) \subseteq \mathscr{M}_{n}(I) \subseteq P_{n}(I)
$$

Proof. The inclusion $\mathscr{M}_{n}(I) \subset P_{n}(I)$ can be shown with the same argument as in [14]. 
To show the inclusion $P_{n+1}(I) \subset \mathscr{M}_{n}(I)$ we take the same steps as in [14]. Take an arbitrary $f \in P_{n+1}(I)$, and choose $0<\lambda_{1}<\lambda_{2}<\cdots<\lambda_{2 n}<a$. Consider

$$
\frac{p(t)}{\pi(t)}=\sum_{k=1}^{2 n} a_{k} \frac{\lambda_{k}}{t+\lambda_{k}},
$$

where $p$ is any polynomial of degree less than or equal to $2 n-1$ (write the class of such polynomials by $\operatorname{Pol}(2 n-1))$ such that $p(t) \geq 0$ for $t>0$ and $p(0)=0$, and

$$
\pi(t)=\prod_{i=1}^{2 n}\left(t+\lambda_{i}\right) .
$$

Then we may show that

$$
\sum_{k=1}^{2 n} a_{k} f\left(\lambda_{k}\right) \geq 0
$$

Note that

$$
a_{k}=\frac{p\left(-\lambda_{k}\right)}{\lambda_{k} \pi^{\prime}\left(-\lambda_{k}\right)}
$$

for $1 \leq k \leq 2 n$, and the polynomials with the above property can be written as

$$
p(t)=t q_{1}(t)^{2}+q_{2}(t)^{2},
$$

where $q_{1}, q_{2} \in \operatorname{Pol}(n-1)$ and $q_{2}(0)=0$. (For example see [12, Lemma 7.6.1].) Hence, because of linearity we only have to consider the two cases $p(t)=t q(t)^{2}$ and $p(t)=q(t)^{2}$ with $q(0)=0$.

When $p(t)=t q(t)^{2}$, we can show the inequality (3) by the same argument as in (i) of the proof in [14, Lemma 1]. We write $0<\lambda_{1}<\lambda_{2}<\cdots<\lambda_{2 n}<a$ as $0<\beta_{1}<\alpha_{1}<\beta_{2}<\cdots<\beta_{n}<\alpha_{n}<a$. When $p(t)=q(t)^{2}$, $q \in \operatorname{Pol}(n-1), q(0)=0$, write

$$
\frac{q^{2}(t)}{\pi(t)}=\sum_{k=1}^{n} y_{k}^{2} \frac{\beta_{k}}{t+\beta_{k}}-\sum_{k=1}^{n} x_{k}^{2} \frac{\alpha_{k}}{t+\alpha_{k}},
$$

where

$$
y_{k}^{2}=\frac{q^{2}\left(-\beta_{k}\right)}{\beta_{k} \pi^{\prime}\left(-\beta_{k}\right)}, \quad x_{k}^{2}=\frac{-q^{2}\left(-\alpha_{k}\right)}{\alpha_{k} \pi^{\prime}\left(-\alpha_{k}\right)} .
$$

We extend $0<\beta_{1}<\alpha_{1}<\beta_{2}<\cdots<\beta_{n}<\alpha_{n}<a$ with $\delta$ and $\omega$ such that

$$
0<\delta<\beta_{1}<\alpha_{1}<\beta_{2}<\cdots<\beta_{n}<\alpha_{n}<\omega<a
$$


and consider

$$
\frac{t}{t+\delta} \cdot \frac{q^{2}(t)}{\pi(t)} \cdot \frac{t+a}{t+\omega}
$$

Note that

$$
\frac{t}{t+\delta} \cdot \frac{q^{2}(t)}{\pi(t)} \cdot \frac{t+a}{t+\omega} \rightarrow \frac{q^{2}(t)}{\pi(t)}
$$

as $\delta \rightarrow 0$ and $\omega \rightarrow a$. By the partial fraction expansion

$$
\frac{t}{t+\delta} \cdot \frac{q^{2}(t)}{\pi(t)} \cdot \frac{t+a}{t+\omega}=-\tilde{x}_{0}^{2} \frac{\delta}{t+\delta}-\sum_{k=1}^{n} \tilde{x}_{k}^{2} \frac{\alpha_{k}}{t+\alpha_{k}}+\sum_{k=1}^{n} \tilde{y}_{k}^{2} \frac{\beta_{k}}{t+\beta_{k}}+\tilde{y}_{n+1}^{2} \frac{\omega}{t+\omega},
$$

where $\tilde{x}_{k}$ and $\tilde{y}_{k}$ are defined similar as $x_{k}$ and $y_{k}$, and

$$
\tilde{x}_{k} \rightarrow x_{k}, \quad \tilde{y}_{k} \rightarrow y_{k}, \quad 1 \leq k \leq n
$$

as $\delta \rightarrow 0$ and $\omega \rightarrow a$. Moreover

$$
\tilde{x}_{0}^{2}=\frac{q^{2}(-\delta)}{\pi(-\delta)} \cdot \frac{a-\delta}{\omega-\delta}=\mathscr{O}\left(\delta^{2}\right), \quad \delta \rightarrow 0,
$$

since $q(0)=0$, and

$$
\tilde{y}_{n+1}^{2}=\frac{q^{2}(-\omega)}{\pi(-\omega)} \cdot \frac{-\omega+a}{\omega-\delta}=\mathscr{O}(-\omega+a), \quad \omega \rightarrow a .
$$

Let $f \in P_{n+1}(I)$. By letting $t=0$ in (4) we have

$$
-\tilde{x}_{0}^{2}-\sum_{k=1}^{n} \tilde{x}_{k}^{2}+\sum_{k=1}^{n} \tilde{y}_{k}^{2}+\tilde{y}_{n+1}^{2}=0 .
$$

Since $\frac{t}{t+\delta} \frac{q^{2}(t)}{\pi(t)} \frac{t+a}{t+\omega} t \geq 0$,

$$
\lim _{t \rightarrow \infty} \frac{t}{t+\delta} \cdot \frac{q^{2}(t)}{\pi(t)} \cdot \frac{t+\alpha}{t+\omega} \cdot t \geq 0 .
$$

Hence

$$
-\tilde{x}_{0}^{2} \delta-\sum_{k=1}^{n} \tilde{x}_{k}^{2} \alpha_{i}+\sum_{k=1}^{n} \tilde{y}_{k}^{2} \beta_{k}+\tilde{y}_{n+1}^{2} \omega \geq 0 .
$$

Since $f \in P_{n+1}(I)$ and (5),

$$
-\tilde{x}_{0}^{2} f(\delta)-\sum_{k=1}^{n} \tilde{x}_{k}^{2} f\left(\alpha_{k}\right)+\sum_{k=1}^{n} \tilde{y}_{k}^{2} f\left(\beta_{k}\right)+\tilde{y}_{n+1}^{2} f(\omega) \geq 0 .
$$


(See Lemma 5.3 and $\left(1^{\prime}\right)$ in [14].)

By the same argument as in (ii) in the proof in [14, Lemma 1], we have

$$
\lim _{\delta \rightarrow 0} \tilde{x}_{0}^{2} f(\delta)=\lim _{\omega \rightarrow a} \tilde{y}_{n+1}^{2} f(\omega)=0 .
$$

Both equalities come from the same proof as in [14, Lemma 1]. Indeed, we consider the following inequality, which is used in [14, Lemma 1]:

$$
\begin{aligned}
- & \frac{\left(c-\beta_{1}\right)^{2}}{\left(\alpha_{1}-\beta_{1}\right)\left(\beta_{2}-\beta_{1}\right)\left(\alpha_{2}-\beta_{1}\right)} f\left(\beta_{1}\right)-\frac{\left(c-\beta_{2}\right)^{2}}{\left(\beta_{1}-\beta_{2}\right)\left(\alpha_{1}-\beta_{2}\right)\left(\alpha_{2}-\beta_{2}\right)} f\left(\beta_{2}\right) \\
& \quad+\frac{\left(c-\alpha_{1}\right)^{2}}{\left(\alpha_{1}-\beta_{1}\right)\left(\beta_{2}-\alpha_{1}\right)\left(\alpha_{2}-\alpha_{1}\right)} f\left(\alpha_{1}\right)+\frac{\left(c-\alpha_{2}\right)^{2}}{\left(\alpha_{2}-\beta_{1}\right)\left(\alpha_{1}-\alpha_{2}\right)\left(\beta_{2}-\alpha_{2}\right)} f\left(\alpha_{2}\right) \geq 0 .
\end{aligned}
$$

This comes from the fact that $f \in P_{2}(I)$ and Lemma 5.3. (See (1') in [14].)

To get the first equality, set $c=\beta_{1}=\frac{\delta}{2}, \alpha_{1}=\delta, \beta_{2}=\frac{a}{4}$, and $\alpha_{2}=\frac{a}{2}$. Then we have

$$
\delta f(\delta) \geq \frac{8}{a}\left\{\left(\frac{a}{2}-\delta\right)\left(\frac{a}{4}-\frac{\delta}{2}\right) f\left(\frac{a}{4}\right)-\left(\frac{a}{2}-\frac{\delta}{2}\right)\left(\frac{a}{4}-\delta\right) f\left(\frac{a}{2}\right)\right\} .
$$

Hence

$$
\lim \inf _{\delta \rightarrow 0} \delta^{2} f(\delta) \geq 0 .
$$

Since $f$ is monotone, $\delta^{2} f(\delta) \leq \delta^{2} f\left(\frac{a}{2}\right)$ for $\delta<\frac{a}{2}$. Then

$$
\lim \sup _{\delta \rightarrow 0} \delta^{2} f(\delta) \leq \lim \sup _{\delta \rightarrow 0} \delta^{2} f\left(\frac{a}{2}\right)=0,
$$

and hence $\lim _{\delta \rightarrow 0} \delta^{2} f(\delta)=0$. Therefore we have

$$
\lim _{\delta \rightarrow 0} \tilde{x}_{0}^{2} f(\delta)=\lim _{\delta \rightarrow 0} \frac{\tilde{x}_{0}^{2}}{\delta^{2}} \delta^{2} f(\delta)=0 \quad\left(\tilde{x}_{0}^{2}=\mathscr{O}\left(\delta^{2}\right)\right) .
$$

To get the second equality set $c=\alpha_{1}=\frac{a}{4}, \beta_{1}=\frac{a}{8}, \beta_{2}=\frac{\omega}{2}$, and $\alpha_{2}=\omega$ with $0<\beta_{1}<\alpha_{1}<\beta_{2}<\alpha_{2}<a$. Then we have

$$
f(\omega) \leq-\frac{\frac{a}{8}\left(\omega-\frac{a}{8}\right) \frac{\omega}{2}}{\omega-\frac{a}{4}} f\left(\frac{a}{8}\right)-\frac{\left(\frac{\omega}{2}-\frac{a}{4}\right)\left(\omega-\frac{a}{8}\right) \frac{\omega}{2}}{\left(\omega-\frac{a}{4}\right)\left(\frac{\omega}{2}-\frac{a}{8}\right) \frac{\omega}{2}} f\left(\frac{\omega}{2}\right) \quad(=h(\omega))
$$

for $\omega \in\left(\frac{a}{2}, a\right)$. Multiplying the above inequality by $(-\omega+a)>0$ for $\omega \in$ $(0, a)$ we obtain that

$$
(-\omega+a) f(\omega) \leq(-\omega+a) h(\omega), \quad \omega \in(0, a) .
$$

Hence

$$
\lim _{\omega \rightarrow a} \sup (a-\omega) f(\omega) \leq 0
$$


On the contrary, since $(a-\omega) f(\omega) \geq(a-\omega) f\left(\frac{a}{2}\right)$ for $\omega \in\left(\frac{a}{2}, a\right)$,

$$
\lim _{\omega \rightarrow a} \inf (a-\omega) f(\omega) \geq 0,
$$

and hence $\lim _{\omega \rightarrow a}(a-w) f(\omega)=0$. Therefore, we have

$$
\lim _{\omega \rightarrow a} \tilde{y}_{n+1}^{2}=\lim _{\omega \rightarrow a} \frac{\tilde{y}_{n+1}^{2}}{(a-\omega)}(a-\omega) f(\omega)=0 \quad\left(\tilde{y}_{n+1}^{2}=\mathcal{O}(a-\omega)\right) .
$$

Hence, we get

$$
-\sum_{k=1}^{n} x_{k}^{2} f\left(\alpha_{k}\right)+\sum_{k=1}^{n} y_{k}^{2} f\left(\beta_{k}\right) \geq 0
$$

and $f \in \mathcal{M}_{n}(I)$.

From the above inclusion property, we have the following characterization of operator monotone functions.

THEOREM 5.5. $f$ is operator monotone on I if and only if

$$
f \in \cap_{n=1}^{\infty} \mathscr{M}_{n}(I) .
$$

\section{Examples}

Let $g_{n}$ be polynomials considered in [5]. In this section, we show that

$$
g_{n} \in P_{n}\left(\left[0, \alpha_{n}\right]\right) \backslash \mathcal{M}_{n}\left(\left[0, \alpha_{n}\right]\right)
$$

for some $\alpha_{n}>0$ and $n=2,3,4,5$, and use Maple to get a numerical value for $\alpha_{n}$.

We believe that $g_{n} \in P_{n}\left(\left[0, \alpha_{n}\right]\right) \backslash \mathcal{M}_{n}\left(\left[0, \alpha_{n}\right]\right)$ for some $\alpha_{n}>0$ and any $n \geq 2$.

\section{1. $g_{2}$ case}

Let $g_{2}(x)=x+\frac{1}{3} x^{3}$, and let $M_{2}\left(g_{2} ; x\right)$ be the matrix function corresponding to $g_{2}$,

$$
M_{2}\left(g_{2} ; x\right)=\left(\begin{array}{cc}
1+x^{2} & x \\
x & \frac{1}{3}
\end{array}\right)
$$

We claim that $g_{2} \in P_{2}\left(\left[0, \alpha_{2}\right]\right)$ for some $\alpha_{2}>\frac{1}{2}$. To this end we have only to show that $M_{2}\left(g_{2} ; x\right)$ is positive definite for all $x \in\left[0, \frac{1}{2}\right]$. Since the determinant $\operatorname{det}\left(M_{2}\left(g_{2} ; x\right)\right)$ is $\frac{1}{3}-\frac{2}{3} x^{2}$, it is easily seen that $\operatorname{det}\left(M_{2}\left(g_{2} ; x\right)\right)>0$ for all $x \in[0,1 / 2]$. Hence $\alpha_{2}>1 / 2$ and $g_{2} \in P\left(\left[0, \alpha_{2}\right]\right)$ by [3, Theorem VIII.V]. 
Next we show that $g_{2} \notin \mathscr{M}\left(\left[0, \alpha_{2}\right]\right)$. We take the polynomial $p(x)=x^{2}$ and

$$
\lambda_{k}=\frac{k}{8}, \quad 1 \leq k \leq 4 .
$$

Since $a_{k}=\frac{p\left(-\lambda_{k}\right)}{\lambda_{k} \pi^{\prime}\left(-\lambda_{k}\right)}$ for $1 \leq k \leq 4$ where $\pi(x)=\prod_{k=1}^{4}\left(x+\lambda_{k}\right)$, we have

$$
\sum_{k=1}^{4} a_{k} g_{2}\left(\lambda_{k}\right)=-\frac{5}{12}<0 .
$$

This implies that $g_{2} \notin M_{2}\left(\left[0, \alpha_{2}\right]\right)$ by Definition 5.1.

\section{2. $g_{3}$ case}

Let $g_{3}(x)=x+1 / 3 x^{3}+1 / 5 x^{5}$, and let $M_{3}\left(g_{3} ; x\right)$ be the corresponding matrix function for $g_{3}$, that is,

$$
M_{3}\left(g_{3} ; x\right)=\left(\begin{array}{ccc}
1+x^{2}+x^{4} & x+2 x^{3} & \frac{1}{3}+2 x^{2} \\
x+2 x^{3} & \frac{1}{3}+2 x^{2} & x \\
\frac{1}{3}+2 x^{2} & x & \frac{1}{5}
\end{array}\right) .
$$

We claim that $g_{3} \in P_{3}\left(\left[0, \alpha_{3}\right]\right)$ for some $\alpha_{3}>\frac{1}{5}$. To get this we have only to show that $M_{3}\left(g_{3} ; x\right)$ is positive definite for all $x \in\left[0, \frac{1}{5}\right]$. The determinants of principal matrices of $M_{3}\left(g_{3} ; x\right)$ are as follows:

$$
\begin{aligned}
\operatorname{det}\left(M_{3}\left(g_{3} ; x\right)_{22}\right) & =\frac{1}{3}+\frac{4}{3} x^{2}-\frac{5}{3} x^{4}-2 x^{6} \\
\operatorname{det}\left(M_{3}\left(g_{3} ; x\right)\right) & =\frac{4}{135}-\frac{11}{15} x^{2}-\frac{7}{5} x^{6},
\end{aligned}
$$

where $M_{3}\left(g_{3} ; x\right)_{22}$ means the $2 \times 2$ upper part of $M_{3}\left(g_{3} ; x\right)$. Then we can conclude that

$$
\operatorname{det}\left(M_{3}\left(g_{3} ; x\right)_{22}\right)>0, \quad \operatorname{det}\left(M_{3}\left(g_{3} ; x\right)\right)>0
$$

for all $x \in\left[0, \frac{1}{5}\right]$. The numerical value of the right end point of the interval has been obtained using computations in Maple (see arXiv.org: math.OA/0606421). We can conclude that $M_{3}\left(g_{3} ; x\right)$ is positive definite for any $x \in\left[0, \frac{1}{5}\right]$. (See $\left[3\right.$, Theorem I.3.3] for example.) Hence $g_{3} \in P_{3}\left(\left[0, \alpha_{3}\right]\right)$ for some $\alpha_{3}>\frac{1}{5}$.

Next we show that $g_{3} \notin \mathscr{M}_{3}\left(\left[0, \alpha_{3}\right]\right)$. Let $p(x)=x^{4}$ and $\lambda_{k}=\frac{k}{30}, 1 \leq k \leq$ 6. Since $a_{k}=\frac{p\left(-\lambda_{k}\right)}{\lambda_{k} \pi^{\prime}\left(-\lambda_{k}\right)}$ for $1 \leq k \leq 6$ and $\pi(x)=\prod_{k=1}^{6}\left(x+\lambda_{k}\right)$, we have

$$
\sum_{k=1}^{6} a_{k} g_{2}\left(\lambda_{k}\right)=-\frac{1897}{7500}<0 .
$$

This implies that $g_{3} \notin \mathscr{M}_{3}\left(\left[0, \alpha_{3}\right]\right)$ by Definition 5.1. 


\section{3. $g_{4}$ case}

Let $g_{4}(x)=x+\frac{1}{3} x^{3}+\frac{1}{5} x^{5}+\frac{1}{7} x^{7}$ and let $M_{4}\left(g_{4} ; x\right)$ be the corresponding matrix function for $g_{4}$, that is,

$$
\begin{aligned}
& M_{4}\left(g_{4} ; x\right) \\
& =\left(\begin{array}{cccc}
1+x^{2}+x^{4}+x^{6} & x+2 x^{3}+3 x^{5} & \frac{1}{3}+2 x^{2}+5 x^{4} & x+5 x^{3} \\
x+2 x^{3}+3 x^{5} & \frac{1}{3}+2 x^{2}+5 x^{4} & x+5 x^{3} & \frac{1}{5}+3 x^{2} \\
\frac{1}{3}+2 x^{2}+5 x^{4} & x+5 x^{3} & \frac{1}{5}+3 x^{2} & x \\
x+5 x^{3} & \frac{1}{5}+3 x^{2} & x & \frac{1}{7}
\end{array}\right) .
\end{aligned}
$$

We claim that $g_{4} \in P_{4}\left(\left[0, \alpha_{4}\right]\right)$ for some $\alpha_{4}>\frac{1}{25}$. To this end we have only to show that $M_{4}\left(g_{4} ; x\right)$ are positive definite for all $x \in\left[0, \frac{1}{25}\right]$. The determinants of all principal matrices of $M_{4}\left(g_{4} ; x\right)$ are as follows:

$$
\begin{aligned}
\operatorname{det}\left(M_{4}\left(g_{4} ; x\right)_{11}\right)= & 1+x^{2}+x^{4}+x^{6}, \\
\operatorname{det}\left(M_{4}\left(g_{4} ; x\right)_{22}\right)= & \frac{1}{3}+\frac{4}{3} x^{2}+\frac{10}{3} x^{4}-\frac{8}{3} x^{6}-5 x^{8}-4 x^{10}, \\
\operatorname{det}\left(M_{4}\left(g_{4} ; x\right)_{33}\right)= & \frac{4}{135}+\frac{4}{15} x^{2}-\frac{10}{3} x^{4}-\frac{118}{15} x^{6}+2 x^{8}-\frac{54}{5} x^{10}-12 x^{12}, \\
\operatorname{det}\left(M_{4}\left(g_{4} ; x\right)\right)= & -\frac{848}{7875} x^{2}+\frac{72}{7} x^{12}-\frac{188}{175} x^{8} \\
& +\frac{72}{35} x^{10}+\frac{16}{23625}+\frac{1472}{7875} x^{4}-\frac{4712}{875} x^{6} .
\end{aligned}
$$

Hence we can conclude that $M_{4}\left(g_{4} ; x\right)$ are positive definite for all $x \in\left[0, \frac{1}{25}\right]$ using Maple, because each of the determinants is strictly positive for any $x \in\left[0, \frac{1}{25}\right]$. The numerical value of the right end point of the interval has been obtained using computations in Maple (see arXiv.org: math.OA/0606421).

Next we claim that $g_{4} \notin \mathscr{M}_{4}\left(\left[0, \alpha_{4}\right]\right)$. We take $p(x)=x^{6}$ and $\lambda_{k}=\frac{k}{200}$ for $1 \leq k \leq 8$. Then by the same argument as in the case of $n=2$ and $n=3$ we have

$$
\sum_{k=1}^{8} a_{k} g_{4}\left(\lambda_{k}\right)=-\frac{33766394903}{56 \times 10^{10}}<0,
$$

and hence $g_{4} \notin \mathscr{M}_{4}\left(\left[0, \alpha_{4}\right]\right)$. 


\section{4. $g_{5}$ case}

Let $g_{5}(x)=x+1 / 3 x^{3}+1 / 5 x^{5}+1 / 7 x^{7}+1 / 9 x^{9}$ and let $M_{5}\left(g_{5} ; x\right)$ be the corresponding matrix function for $g_{5}$, that is,

$$
\begin{aligned}
& M_{5}\left(g_{5} ; x\right) \\
& =\left(\begin{array}{ccc}
1+x^{2}+x^{4}+x^{6}+x^{8} & x+2 x^{3}+3 x^{5}+4 x^{7} & \frac{1}{3}+2 x^{2}+5 x^{4}+\frac{28}{3} x^{6} \\
x+2 x^{3}+3 x^{5}+4 x^{7} & \frac{1}{3}+2 x^{2}+5 x^{4}+\frac{28}{3} x^{6} & x+5 x^{3}+14 x^{5} \\
\frac{1}{3}+2 x^{2}+5 x^{4}+\frac{28}{3} x^{6} & x+5 x^{3}+14 x^{5} & \frac{1}{5}+3 x^{2}+14 x^{4} \\
x+5 x^{3}+14 x^{5} & \frac{1}{5}+3 x^{2}+14 x^{4} & x+\frac{28}{3} x^{3} \\
\frac{1}{5}+3 x^{2}+14 x^{4} & x+\frac{28}{3} x^{3} & \frac{1}{7}+4 x^{2} \\
x+5 x^{3}+14 x^{5} & \frac{1}{5}+3 x^{2}+14 x^{4} \\
\frac{1}{5}+3 x^{2}+14 x^{4} & x+\frac{28}{3} x^{3} \\
x+\frac{28}{3} x^{3} & \frac{1}{7}+4 x^{2} \\
\frac{1}{7}+4 x^{2} & x \\
x & \frac{1}{9}
\end{array}\right) .
\end{aligned}
$$

We claim that $g_{5} \in P_{3}\left(\left[0, \alpha_{5}\right]\right)$ for some $\alpha_{5}>\frac{1}{125}$. To this end we have only to show that all principal matrices of $M_{5}\left(g_{5} ; x\right)$ are positive definite for all $x \in\left[0, \frac{1}{125}\right]$. The determinants of principal matrices of $M_{5}\left(g_{5} ; x\right)$ are as follows:

$$
\begin{aligned}
\operatorname{det}\left(M_{5}\left(g_{5} ; x\right)_{11}\right)=1 & +x^{2}+x^{4}+x^{6}+x^{8}, \\
\operatorname{det}\left(M_{5}\left(g_{5} ; x\right)_{22}\right)=\frac{1}{3} & +\frac{4}{3} x^{2}+\frac{10}{3} x^{4}+\frac{20}{3} x^{6}-\frac{10}{3} x^{8} \\
& -\frac{26}{3} x^{10}-\frac{29}{3} x^{12}-\frac{20}{3} x^{14}, \\
\operatorname{det}\left(M_{5}\left(g_{5} ; x\right)_{33}\right)=\frac{4}{15} x^{2}+\frac{4}{3} x^{4}-\frac{82}{9} x^{6}-\frac{97}{3} x^{8}-\frac{656}{15} x^{10} & +\frac{613}{45} x^{12}-42 x^{14}-\frac{242}{3} x^{16}-\frac{1540}{27} x^{18}+\frac{4}{135}, \\
\operatorname{det}\left(M_{5}\left(g_{5} ; x\right)_{44}\right)=\frac{256}{23625} x^{2}-\frac{18824}{23625} x^{4}-\frac{7136}{2625} x^{6}+\frac{5588}{875} x^{8}+\frac{16}{23625}+\frac{6776}{27} x^{20} & -\frac{137576}{1575} x^{10}-\frac{254962}{1575} x^{12}+\frac{44}{3} x^{14}-\frac{2728}{945} x^{16}+\frac{6776}{27} x^{18}, \\
\operatorname{det}\left(M_{5}\left(g_{5} ; x\right)\right)=- & \frac{34256}{10418625} x^{2}+\frac{69212}{243} x^{20}+\frac{20251814}{138915} x^{12}+\frac{1024}{260465625} \\
& +\frac{1424236}{694575} x^{8}-\frac{284372}{138915} x^{10}+\frac{216592}{10418625} x^{4}+\frac{173030}{1701} x^{18} \\
& -\frac{644930}{11907} x^{16}-\frac{1213916}{694575} x^{6}+\frac{1617407}{27783} x^{14} .
\end{aligned}
$$

Hence we can conclude that $M_{5}\left(g_{5} ; x\right)$ are positive definite for all $x \in\left[0, \frac{1}{125}\right]$. The numerical value of the right end point of the interval has been obtained 
using computations in Maple (see arXiv.org: math.OA/0606421).

Next we claim that $g_{5} \notin \mathscr{M}_{5}\left(\left[0, \alpha_{5}\right]\right)$. As in the case of $g_{5}$, we shall find a polynomial $p$, and positive number $\lambda_{1}, \lambda_{2}, \lambda_{3}, \lambda_{4}, \lambda_{5}, \lambda_{6}, \lambda_{7}, \lambda_{8}, \lambda_{9}, \lambda_{10}$ in $\left[0, \frac{1}{125}\right]$ such that $\sum_{k=1}^{10} a_{k} g_{5}\left(\lambda_{k}\right)<0$, where $a_{k}=\frac{p\left(-\lambda_{k}\right)}{\lambda_{k} \pi^{\prime}\left(-\lambda_{k}\right)}$ and $\pi(x)=$ $\prod_{j=1}^{10}\left(x+\lambda_{j}\right)$.

We take $p(x)=x^{6}$ and $\lambda_{k}=\frac{k}{1250}$ for $1 \leq k \leq 10$. Then by the argument as in the case of $n=2,3$, 4 we have

$$
\sum_{k=1}^{10} a_{k} g_{5}\left(\lambda_{k}\right)=-\frac{33848952554021}{3845214843750000}<0,
$$

and hence $g_{5} \notin M_{5}\left(\left[0, \alpha_{5}\right]\right)$.

\section{Comments}

Motivated by results on operator monotone and matrix monotone functions and their relation to $C^{*}$-algebras [4], [5], [9], [10], [11], [16], [14], and the monotonicity gap inclusion results and the $C^{*}$-algebraic version of interpolation spaces obtained in [1], we feel that the related problem of a $C^{*}$-algebraic interpretation and perhaps a $C^{*}$-algebraic generalization of the spaces $\mathscr{M}_{n}$ is of interest.

\section{REFERENCES}

1. Ameur, Y., Kaijser, S., Silvestrov, S., Interpolation classes and matrix monotone functions, to appear in J. Operator Theory.

2. Curto, R., Fialkow, L. A., Recursiveness, positivity, and truncated moment problems, Houston J. Math. 17, 4 (1991), 603-635.

3. Donoghue Jr., W. F., Monotone Matrix Functions and Analytic Continuation, Springer-Verlag, Berlin Heidelberg New York, 1974.

4. Ji, G., Tomiyama, J., On characterizations of commutativity of $C^{*}$-algebras, Proc. Amer. Math. Soc. 131 (2003), no. 12, 3845-3849.

5. Hansen, F., Ji, G., Tomiyama, J., Gaps between classes of matrix monotone functions, Bull. London Math. Soc. 36 (2004), 53-58.

6. Hiai, F., Yanagi, K., Hilbert Spaces and Linear Operators, Makino Pub. Ltd. 1995.

7. Löwner, C., Über monotone Matrixfunktionen, Math. Z. 38 (1934), 177-216.

8. Nayak, S., Monotone matrix functions of successive orders, Proc. Amer. Math. Soc. 132 (2004), no. 1, 33-35.

9. Ogasawara, T., A theorem on operator algebras, J. Sci. Hiroshima Univ. 18 (1955), 307-309.

10. Osaka, H., Silvestrov, S., Tomiyama, J., Monotone operator functions on $C^{*}$-algebras, Internat. J. Math. 16 (2005), no. 2, 181-196.

11. Pedersen, G. K., $C^{*}$-algebras and their Automorphism Groups, Academic Press, 1979.

12. Persson, T., Operator monotone functions, dynamical systems and non-commutative operator algebras, Master thesis, 2001, Lund. 
13. Horn, R. A., and Johnson, C. R., Topics in Matrix Analysis, Cambridge University Press, Cambridge, 1994.

14. Sparr, G., A new proof of Löwner's theorem on monotone matrix functions, Math. Scand. 47, 2 (1980), 266-274.

15. Wigner, E. P., von Neumann, J., Significance of Loewners theorem in the quantum theory of collisions, Ann. of Math. (2) 59 (1954), 418-433.

16. Wu, W., An order characterization of commutativity for $C^{*}$-algebras, Proc. Amer. Math. Soc. 129 (2001), 983-987.

\author{
DEPARTMENT OF MATHEMATICAL SCIENCES \\ RITSUMEIKAN UNIVERSITY \\ KUSATSU \\ SHIGA 525-8577 \\ JAPAN \\ E-mail: osaka@se.ritsumei.ac.jp \\ TOKYO METROPOLITAN UNIVERSITY \\ 201 11-10 NAKANE 1-CHOME \\ MEGURO-KU \\ TOKYO \\ JAPAN \\ E-mail: jtomiyama@fc.jwu.ac.jp
}

CENTRE FOR MATHEMATICAL SCIENCES LUND UNIVERSITY

BOX 118, SE-22100 LUND

SWEDEN

E-mail: sergei.silvestrov@math.lth.se 